\title{
Elastic scattering of positrons and electrons by argon
}

\author{
Sultana N. Nahar and J. M. Wadehra \\ Department of Physics and Astronomy, Wayne State University, Detroit, Michigan 48202
}

(Received 15 September 1986)

\begin{abstract}
Differential and integrated cross sections for the elastic scattering of low-and intermediate-energy (3-300 eV) positrons and electrons by argon atoms are calculated. Higher transport cross sections, representing moments of $1-(\cos \theta)^{n}$, for these systems are also obtained for $n=1-4$. Model potentials are used to represent the interactions between positrons or electrons and argon atoms. For each impact energy, the phase shifts of the lower partial waves are obtained exactly by numerical integration of the radial equation. The Born approximation is used to obtain the contribution of the higher partial waves to the scattering amplitude. The phase shifts of the seven lowest partial waves are tabulated for various impact energies of positrons and electrons.
\end{abstract}

\section{INTRODUCTION}

Since the pioneer work of Ramsauer, ${ }^{1}$ the study of electron scattering by noble gases has been of considerable theoretical and experimental interest. Observations and calculations of both the total collisional as well as elastic differential and integrated cross sections for the scattering of electrons by rare-gas atoms have been made. In particular, low-energy total collisional cross sections, for electron scattering, exhibit a Ramsauer-Townsend (RT) minimum for $A r, K r$, and $X e$. Observations of the scattering of positrons by rare-gas atoms are relatively recent. "First generation experiments" on positron scattering included observations of the total collisional cross sections. ${ }^{2}$ It was then observed that the scattering of positrons by only the lighter rare-gas atoms $\mathrm{He}, \mathrm{Ne}$, and possibly Ar, exhibited the RT minimum. Thus argon may play a unique role in exhibiting the $\mathrm{RT}$ minimum for the scattering of both the electrons and the positrons. With the advent of high-intensity positron beams, it has now become feasible to carry out "second generation experiments" for measurements of angular distribution of positrons elastically scattered by rare-gas atoms. The impetus for the present paper is provided by the recent measurement ${ }^{3}$ of differential cross sections for elastic scattering of intermediate-energy positrons by argon atoms. In the present calculations of elastic scattering of electrons and positrons by argon, an attempt is made to use as similar (within physical consistency) potential for the two projectiles as is possible.

Elastic and total collisional scattering of electrons by argon has been given considerable attention, both experimentally as well as theoretically, during the past sixty years. ${ }^{1,4-55} \mathrm{Webb}^{4}$ has summarized the electron-argon scattering results prior to 1935 . The later experiments on integrated elastic and total collisional cross sections were performed by Aberth et al. ${ }^{5}(15-25 \mathrm{eV})$, Golden and Bandel $^{6}(0.1-21.6 \mathrm{eV})$, Kauppila et al. ${ }^{7}(1.5-15.7 \mathrm{eV})$, Wagenaar and de Heer $^{8}(25-750 \mathrm{eV})$, Wagenaar and de Heer $^{9}(20-100 \mathrm{eV})$, Kauppila et al. ${ }^{10}(15-800 \mathrm{eV})$, Nickel et al. ${ }^{11}(4-300 \mathrm{eV})$, Jost et al. ${ }^{12}(0.08-54.423 \mathrm{eV})$, Ferch et al. ${ }^{13}(0.08-20 \mathrm{eV})$, Charlton et al. ${ }^{14}(2-50 \mathrm{eV})$, and Gus'kov et al. ${ }^{15}(0.025-1.0 \mathrm{eV})$. The differential cross sections (DCS) for the elastic scattering of electrons by argon have been measured by $\mathrm{Mehr}^{16}(5-1000 \mathrm{eV}$, $\left.20^{\circ}-155^{\circ}\right)$, Schackert ${ }^{17}\left(40-150 \mathrm{eV}, 30^{\circ}-150^{\circ}\right)$, Bromberg $^{18}\left(200-700 \mathrm{eV}, 2^{\circ}-30^{\circ}\right)$, Williams and Willis ${ }^{19}$ $\left(20-400 \mathrm{eV}, 20^{\circ}-150^{\circ}\right)$, Jansen et al. ${ }^{20}(100-3000 \mathrm{eV}$, $\left.5^{\circ}-55^{\circ}\right)$, Lewis et al..$^{2 !}\left(15-200 \mathrm{eV}, 20^{\circ}-140^{\circ}\right)$, Vušković and Kurepa ${ }^{22}\left(60-150 \mathrm{eV}, 5^{\circ}-150^{\circ}\right)$, DuBois and Rudd ${ }^{23}$ $\left(20-800 \mathrm{eV}, 2^{\circ}-150^{\circ}\right)$, Gupta and $\operatorname{Rees}^{24,25}(100 \mathrm{eV}$, $\left.10^{\circ}-150^{\circ}\right)$, Williams ${ }^{26}\left(0.5-20 \mathrm{eV}, 15^{\circ}-150^{\circ}\right)$, Srivastava et $a .^{27}\left(3-100 \mathrm{eV}, 20^{\circ}-135^{\circ}\right)$, Andrick ${ }^{28}(1-20 \mathrm{eV}$, $\left.0^{\circ}-180^{\circ}\right)$, Qing et al. ${ }^{29}\left(10-50 \mathrm{eV}, 40^{\circ}-110^{\circ}\right)$, and Filipović $^{30}\left(10-100 \mathrm{eV}, 20^{\circ}-150^{\circ}\right)$. From experimental angular distribution measurements, integrated elastic cross sections have also been calculated in some cases. Semiempirical cross sections for elastic and inelastic scattering of electrons from argon in the energy range 20 to $3000 \mathrm{eV}$ have been obtained by de Heer et al. ${ }^{31}$ Momentum transfer cross sections for electron-argon scattering have been measured or derived from experimental parameters by McPherson et al. ${ }^{32}(0.08-4 \mathrm{eV})$, Golovanivsky and Kabilan $^{33}(0.005-0.6 \mathrm{eV})$, and Haddad and $O^{\prime} \mathbf{M a l l e y}^{34}$ $(0-4 \mathrm{eV})$. Theoretical studies of electron- or positronargon scattering are characterized by the method used as well as by the potential used in the calculations. Theoretical calculations for electron scattering by argon have been performed by Walker ${ }^{35}$ and by Fink and Yates ${ }^{36}$ using the relativistic approximation; Thompson, ${ }^{37}$ Garbaty and LaBahn, ${ }^{38}$ Yau et al. ${ }^{39}$ and Dasgupta and Bhatia ${ }^{40}$ using the polarized orbital method; Fon et al., ${ }^{41}$ and Bell et $a .^{42}$ using the $R$-matrix method; Pindzola and Kelly, ${ }^{43}$ Amusia et al., ${ }^{44}$ McCarthy et al., ${ }^{45}$ Joachain et al., 46,47 and Staszewska et al. ${ }^{48}$ using optical model potentials; Berg et $a l^{49}$ and Datta et al. ${ }^{\text {so }}$ using model potentials; Khare and Shobha ${ }^{51}$ using the first Born approximation; McEachran and Stauffer ${ }^{52}$ using adiabatic exchange approximation; and Haberland et al. ${ }^{53}$ using Kohn-Shamtype one-particle theory. Momentum transfer cross sections for electron-argon scattering are obtained by Frost and Phelps ${ }^{54}$ from transport coefficients and by Milloy et $a l .{ }^{55}$ by a swarm technique.

All measurements of positron scattering by argon have 
been made in the last decade or so. Measurements of total collisional cross sections for positron scattering from argon include those by Canter et al. ${ }^{56}(2-400 \mathrm{eV})$, Jaduszliwer and Paul $^{57}(4-9 \mathrm{eV})$, Kauppila et al. ${ }^{58}$ $(0.4-18 \mathrm{eV})$, Coleman et al. ${ }^{59}(2-960 \mathrm{eV})$, Griffith et al. ${ }^{60}(30-800 \mathrm{eV})$, Tsai et al. ${ }^{61}(25-300 \mathrm{eV})$, Brenton et al. ${ }^{62}(200-1000 \mathrm{eV})$, Sinapius et al. ${ }^{63}(1-6 \mathrm{eV})$, Coleman et al. ${ }^{64}(2-50 \mathrm{eV})$, and Kauppila et al. ${ }^{10}(15-800$ eV). A recent summary of positron-gas scattering is given by Stein and Kauppila. ${ }^{65}$ Measurements of the angular distribution for positron-argon elastic scattering have been made by Coleman and $\mathrm{McNutt}^{66}\left(2.2-8.7 \mathrm{eV}, 20^{\circ}-60^{\circ}\right)$ and by Hyder et al. ${ }^{3}\left(100-300 \mathrm{eV}, 30^{\circ}-135^{\circ}\right)$. On the theoretical side, positron-argon scattering calculations have been carried out by Joachain et al. ${ }^{46}$ and Khare et al. ${ }^{67}$ using optical model potentials; McEachran et al. ${ }^{68}$ and McEachran and Stauffer ${ }^{69}$ using the polarized orbital method; and Datta et al. ${ }^{50}$ and Arifov and Zhuravleva $^{70}$ using a model potential. In the present study of elastic scattering of low- and intermediate-energy positrons and electrons from argon atoms, model static and Buckingham-type polarization potentials for the positron scattering and the same static (albeit with opposite sign) and polarization potentials along with an exchange potential for electron scattering have been used. The results of the present calculation are compared with the recent experimental observations of positrons and electrons elastically scattered from argon.

\section{THEORY}

Consider a projectile of charge $e_{p}$, with laboratoryframe impact energy $E$, being scattered elastically by a target with central potential $V(r)$. The scattering can be described by the radial part, $u_{l}(r)$, of the $l$ th partial wave of the wave function which satisfies (in atomic units)

$$
\left[\frac{d^{2}}{d r^{2}}-\frac{l(l+1)}{r^{2}}+2 \mu[E-V(r)]\right) u_{l}(r)=0 .
$$

Here $\mu$ is the reduced mass of the system. The asymptotic form of the radial part of the wave function is

$$
u_{l}(r) \underset{r \rightarrow \infty}{\rightarrow} k r\left[j_{l}(k r)-\left(\tan \delta_{l}\right) n_{l}(k r)\right]
$$

where $k^{2}=2 \mu E . j_{l}$ and $n_{l}$ are the spherical Bessel functions of the first and the second kind, respectively. (For their numerical evaluation, see Appendix A.) For positron and electron impact, $\mu=1 . \delta_{l}$ is the energydependent phase shift caused by the potential $V(r)$. From the values of the wave function at two adjacent points $r$ and $r+h(h \ll r)$, in the asymptotic domain, one can extract the phase shift

$\tan \delta_{l}=-\frac{(r+h) u_{l}(r) j_{l}(k(r+h))-r u_{l}(r+h) j_{l}(k r)}{r u_{l}(r+h) n_{l}(k r)-(r+h) u_{l}(r) n_{l}(k(r+h))}$.

Various phase shifts are used to obtain the scattering amplitude as

$$
f(\theta)=\frac{1}{2 i k} \sum_{l=0}^{\infty}(2 I+1)\left(e^{2 i \delta_{l}}-1\right) P_{l}(\cos \theta),
$$

where $\theta$ is the scattering angle. Equation ( 1 ) is solved by using the Numerov procedure and the first $L$ phase shifts are obtained exactly. $L$ depends on the energy of the incident projectile. For large $l(>L)$ the exact phase shifts $\delta_{l}$ are approximately equal to the Born phase shifts $\delta_{B l}$,

$\exp \left(i \delta_{B l}\right) \sin \delta_{B l} \equiv T_{B l}=-2 k \int_{0}^{\infty} r^{2} j_{l}^{2}(k r) V(r) d r$.

The typical values of $L$ corresponding to the impact energies of 3 and $300 \mathrm{eV}$ are 4 and 20 , respectively.

The infinite sum in Eq. (4) is then approximated by

$$
\begin{aligned}
f(\theta)=\frac{1}{2 i k} \sum_{l=0}^{L} & (2 l+1)\left[\exp \left(2 i \delta_{l}\right)-1-\exp \left(2 i \delta_{B l}\right)+1\right] \\
& \times P_{l}(\cos \theta)+f_{B}(\theta),
\end{aligned}
$$

where $f_{B}$ is the scattering amplitude in the Born approximation. For a spherically symmetric potential $V(r)$,

$$
\begin{aligned}
f_{B}(\theta) & =\frac{1}{2 i k} \sum_{l=0}^{\infty}(2 l+1)\left[\exp \left(2 i \delta_{B l}\right)-1\right] P_{l}(\cos \theta) \\
& =-2 \int_{0}^{\infty} r^{2} \frac{\sin (g r)}{q r} V(r) d r
\end{aligned}
$$

where $q=2 k \sin (\theta / 2)$ is the momentum transfer. The differential and the integrated elastic cross sections are

$$
\begin{aligned}
& \frac{d \sigma}{d \Omega}=|f(\theta)|^{2}, \\
& \sigma_{I}=2 \pi \int_{0}^{\pi}\left[\frac{d \sigma}{d \Omega}\right] \sin \theta d \theta .
\end{aligned}
$$

The transport cross sections (including the momentum transfer, $n=1$, cross section) are

$$
\sigma^{(n)}=2 \pi \int_{0}^{\pi}\left(1-\cos ^{n} \theta\right)\left(\frac{d \sigma}{d \Omega}\right) \sin \theta d \theta
$$

TABLE I. Range of the values and the value used for the parameter $d$ (in units of $a_{0}$ ) for various impact energies.

\begin{tabular}{ccc}
\hline \hline & $\begin{array}{c}d \text { (in units of } a_{0} \text { ) } \\
\text { Value used }\end{array}$ \\
\hline 3 & Range of values & 1.39 \\
5 & $1.38-1.4$ & 1.35 \\
10 & $1.25-1.45$ & 1.7 \\
15 & $1.65-1.8$ & 1.6 \\
20 & $1.6-1.8$ & 1.75 \\
30 & $1.7-2.75$ & 1.65 \\
40 & $1.3-1.7$ & 1.6 \\
50 & $1.35-1.7$ & 1.5 \\
75 & $1.3-2.0$ & 2.0 \\
100 & $1.65-2.35$ & 2.0 \\
150 & $1.5-2.5$ & 2.0 \\
200 & $1.5-3.0$ & 2.0 \\
250 & $1.5-3.0$ & 2.0 \\
300 & $1.5-3.0$ & 2.0 \\
\hline
\end{tabular}


The optical theorem

$$
\sigma_{I}=\frac{4 \pi}{k} \operatorname{Im} f(0)
$$

is used as a self-consistent check on the present calculations. In the present calculations of elastic scattering of electrons and positrons by argon atoms, the potentials used are

$$
\begin{aligned}
V(r) & =V_{s}(r)+V_{p}(r) \text { for positron impact } \\
& =V_{s}(r)+V_{p}(r)+V_{\text {ex }}(r) \text { for electron impact } .
\end{aligned}
$$

Here $V_{s}(r)$ is the static potential of the target atom, obtained by averaging over the motion of the target electrons:

$$
\begin{aligned}
V_{s}(\mathbf{r})=\frac{Z e_{p}}{r}-\sum_{i=1}^{Z} e_{p} \int & \left|\Psi\left(\mathbf{r}_{1}, \mathbf{r}_{2}, \ldots, \mathbf{r}_{Z}\right)\right|^{2} \\
& \times \frac{1}{\left|\mathbf{r}-\mathbf{r}_{l}\right|} d \mathbf{r}_{1} \cdots d \mathbf{r}_{Z},
\end{aligned}
$$

where $Z$ is the nuclear charge of the target atom. $\Psi\left(r_{1}, \ldots, r_{Z}\right)$ is the antisymmetrized Hartree-Fock wave function of the target and is expanded in terms of the Slater-type orbitals:

$$
\begin{aligned}
\Phi_{\lambda p}(\mathbf{r}) & =\sum_{i=1}^{M} A(\lambda, p, i) r^{n(p, i)-1} \exp [-\zeta(p, i) r] Y_{l m}(\hat{\mathrm{r}}) \\
& \equiv \phi_{\lambda_{p}}(r) Y_{l m}(\hat{\mathrm{r}})
\end{aligned}
$$

with

$$
A(\lambda, p, i)=c(\lambda, p, i)[2 \zeta(p, i)]^{n(p, i)+1 / 2} /\{[2 n(p, i)] !\}^{1 / 2} .
$$

The values of $c(\lambda, p, i), \zeta(p, i)$, and $n(p, i)$ are taken from the tables of Clementi and Roetti. ${ }^{71}$ Defining, for convenience, $v=n(p, i)+n(p, j), z=\zeta(p, i)+\zeta(p, j), \quad a=A(\lambda, p, i) A(\lambda, p, j) v !, \quad s=z^{-v-1}, \quad m=[1 /(t+1) !-1 /(t ! v)] / z^{v-t}$,

where $i, j$, and $t$ are integers, the static potential for argon atom is given by

$$
V_{s}(r)=e_{p} \sum_{\lambda=1}^{N} \sum_{p=0}^{\lambda-1} N_{\lambda p} \sum_{i=1}^{M} \sum_{j=1}^{M} a \exp (-z r)\left(\frac{s}{r}+\sum_{t=0}^{v-2} m r^{t}\right),
$$

where $N$ is the number of occupied shells in the atom and $N_{\lambda_{p}}$ is the number of electrons in the orbital $(\lambda, p)$. $V_{p}$ is taken to be a model polarization potential of the Buckingham type,

$$
V_{p}(r)=-\frac{1}{2} \alpha r^{2} /\left(r^{2}+d^{2}\right)^{3},
$$

where $\alpha$ is the static dipole polarizability. $d$ is an energy-dependent adjustable parameter determined by fitting the calculated differential and integrated cross sections for the elastic scattering of electrons by argon atoms with the experimental values of the same at a particular energy. The same value of $d$ is then used for positron-argon scattering calculations at that energy. The values of the parameter $d$ for various impact energies are given in Table $\mathbf{I}$. Khare et al., ${ }^{67,72}$ who have used a very similar polarization potential, have expressed the parameter $d$ as a linear function of $k$ in their work. The exchange potential, $V_{e x}(r)$, for a closed-shell atom is taken to be ${ }^{73}$

$$
V_{\mathrm{ex}}(r)=\frac{1}{2}\left[\left[E-V_{D}(r)\right]-\left[\left[E-V_{D}(r)\right]^{2}+\sum_{\lambda=1}^{N} \sum_{p=0}^{\lambda-1} N_{\lambda_{p}}\left|\phi_{\lambda_{p}}(r)\right|^{2}\right]^{1 / 2}\right],
$$

where $V_{D}$ is the direct interaction potential, namely, $V_{D}=V_{s}+V_{p} . \phi_{\lambda p}(r)$ is the radial part of the Slater-type orbital as in Eq. (15). $V_{\text {ex }}(r)$ is a shorter range and much weaker potential than the static potential. Hence it is excluded from the computation of the phase shifts of higher partial waves using the Born approximation. For the polarization potential, the integral for the lth Born phase shift, Eq. (5), defined by $T_{p B I}$, is (for its derivation see Appendix B)

$$
\begin{aligned}
T_{p B l}=\frac{1}{4} \alpha k^{2}\{ & -(2 l+3) i_{l+1}(k d) k_{l}(k d)+\pi(2 l+3) /\left(4 k^{2} d^{2}\right) \\
& \left.-[k d+(2 l+1)(2 l+3) /(2 k d)] i_{l}(k d) k_{l}(k d)+k d i_{l+1}(k d) k_{l+1}(k d)\right\},
\end{aligned}
$$

where $i_{l}$ and $k_{l}$ are the modified spherical Bessel functions of the first and the third kind, respectively. (For their numerical evaluation see Appendix A.) The Born amplitude, Eq. (7), for the polarization potential defined above, is

$$
f_{B p}=\pi \alpha(3-q d) \exp (-q d) /(16 d) .
$$

For the static potential, the lth Born phase shift, Eq. (5), defined to be $T_{s B l}$, is (for details see Appendix C)

$$
T_{s B l}=-\frac{e_{p}}{k} \sum_{\lambda=1}^{N} \sum_{p=0}^{\lambda-1} N_{\lambda p} \sum_{i=1}^{M} \sum_{j=1}^{M} a\left[s Q_{l}\left[1+\frac{z^{2}}{2 k^{2}}\right]+\sum_{t=0}^{v-2}(-1)^{t+1} m \frac{d^{t+1}}{d z^{t+1}} Q_{l}\left[1+\frac{z^{2}}{2 k^{2}}\right]\right],
$$

where $v, z, a, s$, and $m$ are defined in (16). $Q_{l}$ is the Legendre function of the second kind. The corresponding Born scattering amplitude, $f_{B s}$, is 


$$
\left.f_{B s}=-2 e_{p} \sum_{\lambda=1}^{N} \sum_{p=0}^{\lambda-1} N_{\lambda p} \sum_{i=1}^{M} \sum_{j=1}^{M} a \mid \frac{s}{z^{2}+q^{2}}+\sum_{t=0}^{v-2}(-1)^{t+1} m \frac{d^{t+1}}{d z^{t+1}} \frac{1}{z^{2}+q^{2}}\right\rfloor .
$$

For a given impact energy, the polarization potential provides the major contribution and the static potential a non-negligible contribution to the phase shifts of higher partial waves especially for the case of positron impact. The reason is that due to the opposite nature of the static and polarization interactions for the positron case, the

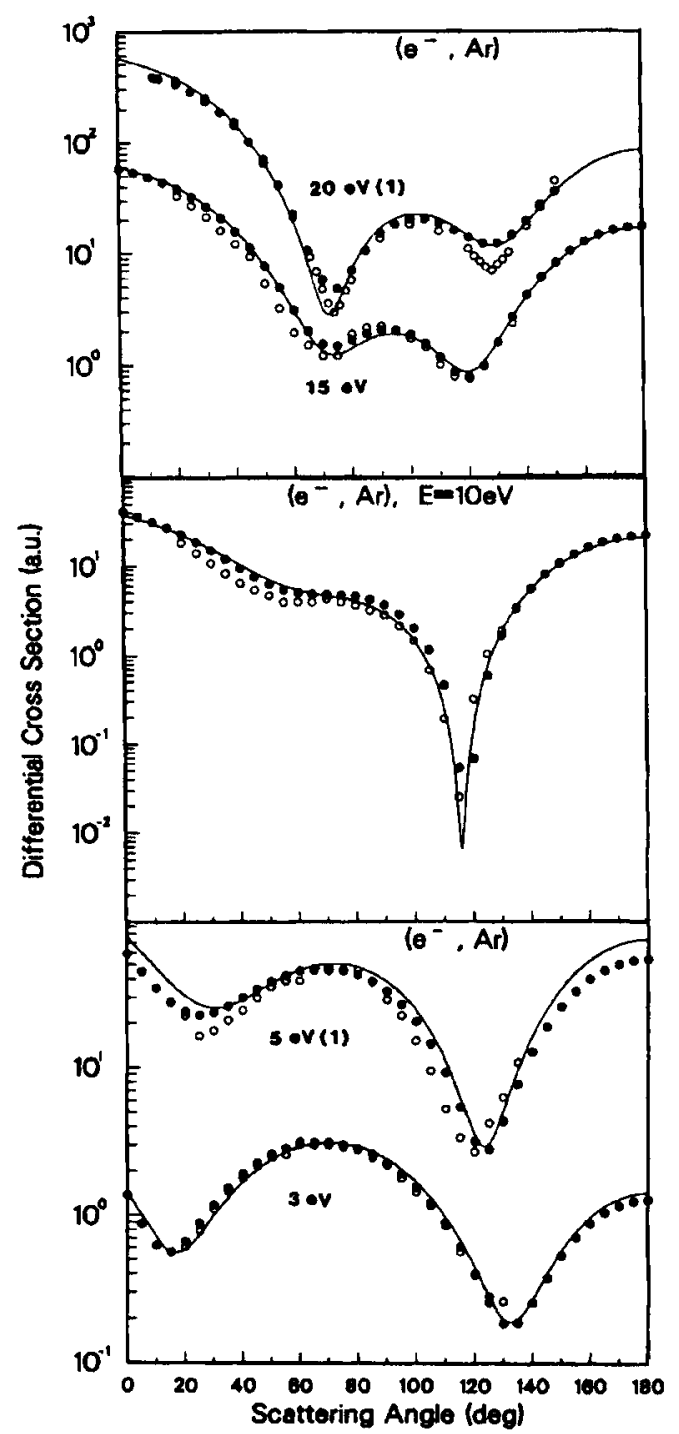

FIG. 1. Differential cross sections for the elastic scattering of electrons by argon at various impact energies. Solid lines are the present theoretical curves. The number in parenthesis following an energy value indicates the power of ten by which the cross section values are multiplied. The experimental values are open circles, Ref. 27 for 3, 5, 10, and $15 \mathrm{eV}$ and Ref. 19 for $20 \mathrm{eV}$; closed circles, Ref. 28 for $3,5,10$, and $15 \mathrm{eV}$ and Ref. 23 for 20 eV. first part of the scattering amplitude obtained from the first $L$ exact phase shifts [see Eq. (6)] is smaller for positron impact than for electron impact and thus the relative contribution of the Born-approximation part with static interaction is more significant for positron impact than for electron impact.

\section{RESULTS AND DISCUSSION}

The differential and integrated cross sections for the elastic scattering of electrons from argon are shown in

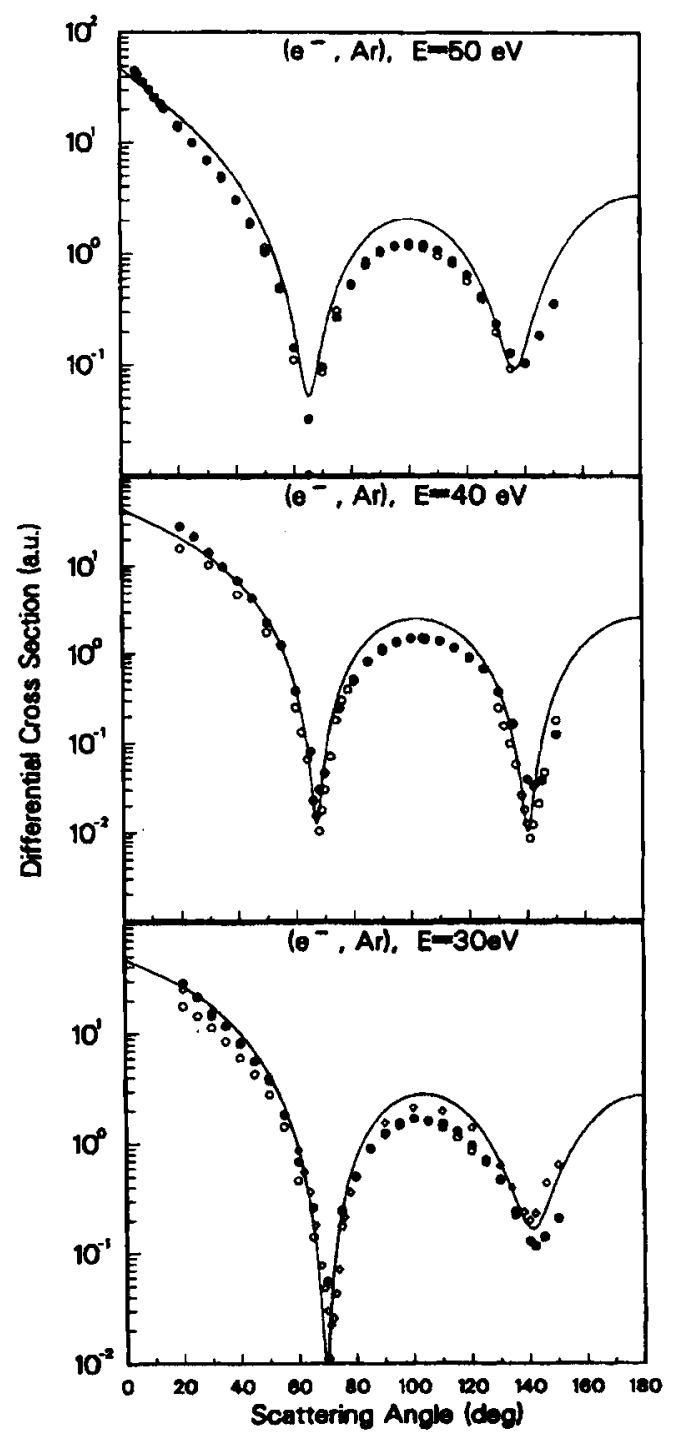

FIG. 2. Same as Fig. 1 except the experimental values are open circles, Ref. 27 for 30 and $50 \mathrm{eV}$ and Ref. 19 for $40 \mathrm{eV}$; closed circles, Ref. 30 for 30 and $40 \mathrm{eV}$ and Ref. 23 for $50 \mathrm{eV}$; diamonds, Ref. 19 for $30 \mathrm{eV}$. 


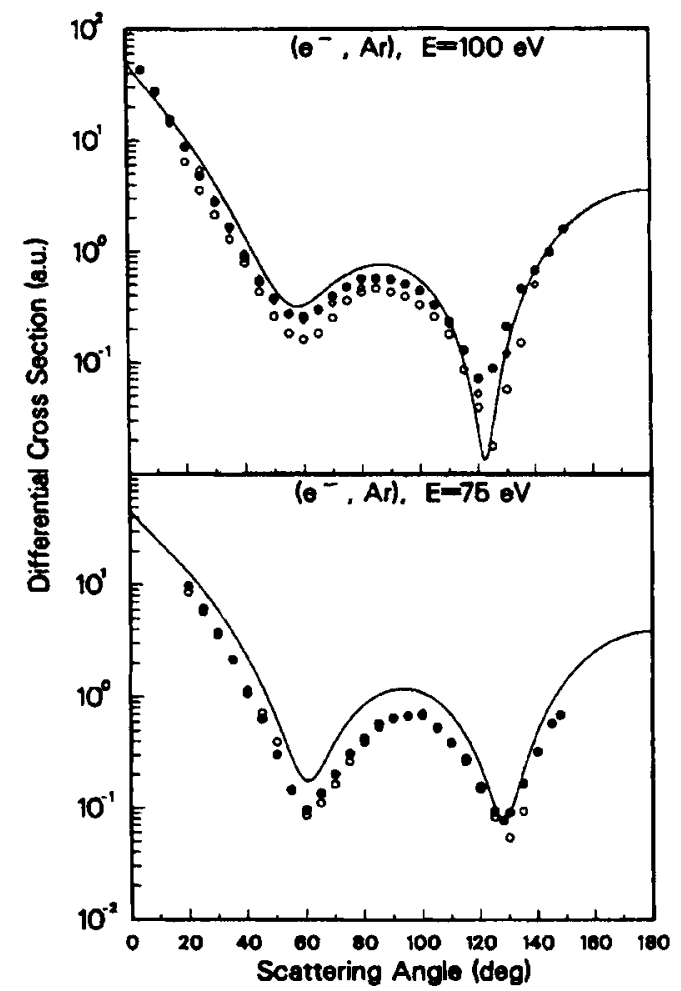

FIG. 3. Same as Fig. 1 except the experimental values are open circles, Ref. 27 for 75 and $100 \mathrm{eV}$; closed circles, Ref. 30 for $75 \mathrm{eV}$ and Ref. 22 for $100 \mathrm{eV}$; diamonds, Ref. 25 for $100 \mathrm{eV}$.

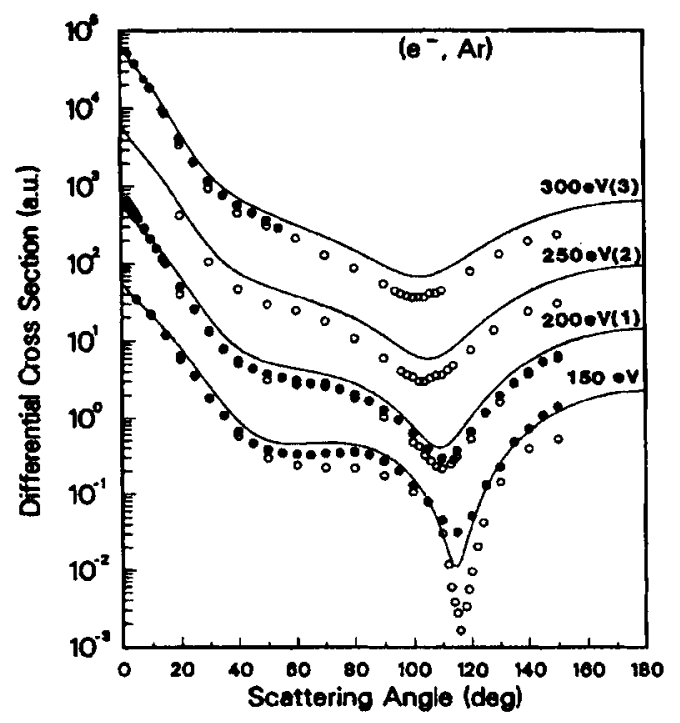

FIG. 4. Same as Fig. 1 except the experimental values are open circles, Ref. 19 for 150, 200, 250, and $300 \mathrm{eV}$; closed circles, Ref. 22 for $150 \mathrm{eV}$, Ref. 23 for $200 \mathrm{eV}$, and Ref. 20 for 300 $\mathrm{eV}$; diamonds, Ref. 18 for $300 \mathrm{eV}$.
Figs. 1-4. The single adjustable parameter $d$ in the polarization potential has been varied, for each impact ener$\mathrm{gy}$, to fit, as closely as possible, the experimentally observed differential as well as integrated cross sections for elastic scattering of electrons from argon. It was noticed that a finite range of values of $d$ could be used for such a fitting procedure. Table I gives the range of values of $d$ along with the final value of $d$ used for computing the present cross sections. It is seen by observing the size of the range of values of $d$ from Table I that the cross sections are more sensitive to the value of $d$ at lower energies

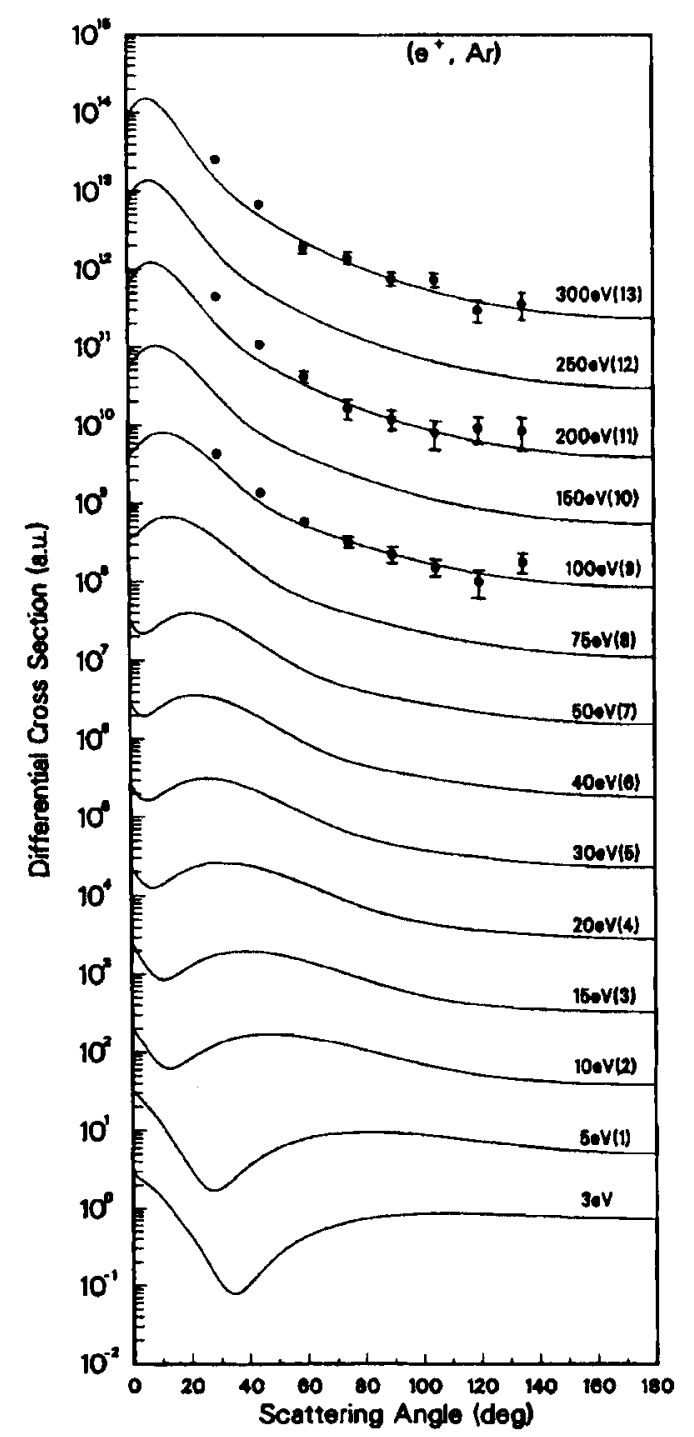

FIG. 5. Differential cross sections for the elastic scattering of positrons by argon at various impact energies. Solid lines are the present theoretical curves. The number in parentheses following an energy value indicates the power of ten by which the cross section values are multiplied. The experimental values are from Ref. 3. 
TABLE II. Differential and integrated cross sections $\left(a_{0}^{2} \mathrm{sr}^{-1}\right)$ for elastic scattering of electrons from argon at $E=3-300 \mathrm{eV}$.

\begin{tabular}{|c|c|c|c|c|c|c|c|}
\hline$\theta(\mathrm{deg})$ & 3 & 5 & 10 & 15 & 20 & 30 & 40 \\
\hline 0 & $0.147[1]^{\mathrm{a}}$ & $0.760[1]$ & $0.377[2]$ & $0.602[2]$ & $0.571[2]$ & $0.472[2]$ & $0.460[2]$ \\
\hline 5 & $0.102[1]$ & $0.608[1]$ & $0.341[2]$ & $0.557[2]$ & $0.523[2]$ & $0.409[2]$ & $0.373[2]$ \\
\hline 10 & 0.717 & $0.481[1]$ & $0.300[2]$ & $0.509[2]$ & $0.476[2]$ & $0.359[2]$ & $0.308[2]$ \\
\hline 15 & 0.560 & $0.379[1]$ & $0.262[2]$ & $0.455[2]$ & $0.426[2]$ & $0.312[2]$ & $0.254[2]$ \\
\hline 20 & 0.585 & $0.311[1]$ & $0.224[2]$ & $0.396[2]$ & $0.371[2]$ & $0.267[2]$ & $0.208[2]$ \\
\hline 25 & 0.742 & $0.271[1]$ & $0.189[2]$ & $0.335[2]$ & $0.314[2]$ & $0.222[2]$ & $0.166[2]$ \\
\hline 30 & $0.101[1]$ & $0.257[1]$ & $0.156[2]$ & $0.274[2]$ & $0.256[2]$ & $0.179[2]$ & $0.129[2]$ \\
\hline 35 & $0.133[1]$ & $0.265[1]$ & $0.127[2]$ & $0.215[2]$ & $0.200[2]$ & $0.138[2]$ & $0.955[1]$ \\
\hline 40 & $0.170[1]$ & $0.291[1]$ & $0.103[2]$ & $0.161[2]$ & $0.149[2]$ & $0.101[2]$ & $0.673[1]$ \\
\hline 45 & $0.207[1]$ & $0.330[1]$ & $0.843[1]$ & $0.115[2]$ & $0.104[2]$ & $0.685[1]$ & $0.440[1]$ \\
\hline 50 & $0.242[1]$ & $0.377[1]$ & $0.699[1]$ & $0.777[1]$ & $0.673[1]$ & $0.422[1]$ & $0.257[1]$ \\
\hline 55 & $0.272[1]$ & $0.424[1]$ & $0.599[1]$ & $0.496[1]$ & $0.391[1]$ & $0.225[1]$ & $0.125[1]$ \\
\hline 60 & $0.295[1]$ & $0.466[1]$ & $0.534[1]$ & $0.302[1]$ & $0.196[1]$ & 0.916 & 0.423 \\
\hline 65 & $0.310[\mathrm{I}]$ & $0.498[1]$ & $0.491[1]$ & $0.187[1]$ & 0.808 & 0.198 & $0.492[-1]$ \\
\hline 70 & $0.315[1]$ & $0.513[1]$ & $0.461[1]$ & $0.134[1]$ & 0.322 & $0.110[-1]$ & $0.619[-1]$ \\
\hline 75 & $0.310[1]$ & $0.510[1]$ & $0.433[1]$ & $0.125[1]$ & 0.341 & 0.238 & 0.368 \\
\hline 80 & $0.295[1]$ & $0.488[1]$ & $0.397[1]$ & $0.140[1]$ & 0.686 & 0.739 & 0.859 \\
\hline 85 & $0.273[1]$ & $0.447[1]$ & $0.350[1]$ & $0.164[1]$ & $0.118[1]$ & $0.137[1]$ & $0.142[1]$ \\
\hline 90 & $0.243[1]$ & $0.390[1]$ & $0.290[1]$ & $0.183[1]$ & $0.168[1]$ & $0.198[1]$ & $0.193[1]$ \\
\hline 95 & $0.208[1]$ & $0.322[1]$ & $0.220[1]$ & $0.187[1]$ & $0.205[1]$ & $0.247[1]$ & $0.231[1]$ \\
\hline 100 & $0.171[1]$ & $0.248[1]$ & $0.145[1]$ & $0.176[1]$ & $0.224[1]$ & $0.275[1]$ & $0.250[1]$ \\
\hline 105 & $0.134[1]$ & $0.177[1]$ & 0.771 & $0.151[1]$ & $0.222[1]$ & $0.279[1]$ & $0.247[1]$ \\
\hline 110 & 0.990 & $0.113[1]$ & 0.252 & $0.121[1]$ & $0.204[1]$ & $0.259[1]$ & $0.223[1]$ \\
\hline 115 & 0.685 & 0.643 & $0.120[-1]$ & 0.951 & $0.174[1]$ & $0.219[1]$ & $0.183[1]$ \\
\hline 120 & 0.446 & 0.358 & 0.150 & 0.864 & $0.144[1]$ & $0.168[1]$ & $0.134[1]$ \\
\hline 125 & 0.281 & 0.311 & 0.741 & $0.106[1]$ & $0.122[1]$ & $0.114[1]$ & $0.843^{\circ}$ \\
\hline 130 & 0.199 & 0.519 & $0.182[1]$ & $0.164[1]$ & $0.117[1]$ & 0.663 & 0.412 \\
\hline 135 & 0.197 & 0.980 & $0.339[1]$ & $0.264[1]$ & $0.137[1]$ & 0.321 & 0.119 \\
\hline 140 & 0.269 & $0.166[1]$ & $0.539[1]$ & $0.407[1]$ & $0.185[1]$ & 0.172 & $0.103[-1]$ \\
\hline 145 & 0.403 & $0.252[1]$ & $0.773[1]$ & $0.586[1]$ & $0.261[1]$ & 0.234 & 0.101 \\
\hline 150 & 0.581 & $0.350[1]$ & $0.103[2]$ & $0.793[1]$ & $0.358[1]$ & 0.495 & 0.375 \\
\hline 155 & 0.781 & $0.451[1]$ & $0.128[2]$ & $0.101[2]$ & $0.469[1]$ & 0.912 & 0.791 \\
\hline 160 & 0.985 & $0.548[1]$ & $0.152[2]$ & $0.122[2]$ & $0.583[1]$ & $0.141[1]$ & $0.128[1]$ \\
\hline 165 & $0.117[1]$ & $0.633[1]$ & $0.173[2]$ & $0.141[2]$ & $0.688[1]$ & $0.192[1]$ & $0.177[1]$ \\
\hline 170 & $0.131[1]$ & $0.700[1]$ & $0.190[2]$ & $0.156[2]$ & $0.773[1]$ & $0.236[1]$ & $0.219[1]$ \\
\hline 175 & $0.141[1]$ & $0.742[1]$ & $0.200[2]$ & $0.166[2]$ & $0.828[1]$ & $0.265[1]$ & $0.247[1]$ \\
\hline 180 & $0.144[1]$ & $0.755[1]$ & $0.204[2]$ & $0.169[2]$ & $0.847[1]$ & $0.275[1]$ & $0.257[1]$ \\
\hline$\sigma_{J}$ & $0.207[2]$ & $0.393[2]$ & $0.763[2]$ & $0.866[2]$ & $0.728[2]$ & $0.511[2]$ & $0.396[2]$ \\
\hline V) & 50 & 75 & 100 & 150 & 200 & 250 & 300 \\
\hline 0 & $0.488[2]$ & $0.474[2]$ & $0.505[2]$ & $0.555[2]$ & $0.595[2]$ & $0.630[2]$ & $0.661[2]$ \\
\hline 5 & $0.374[2]$ & $0.332[2]$ & $0.334[2]$ & $0.337[2]$ & $0.341[2]$ & $0.344[2]$ & $0.347[2]$ \\
\hline 10 & $0.293[2]$ & $0.244[2]$ & $0.232[2]$ & $0.218[2]$ & $0.208[2]$ & $0.201[2]$ & $0.194[2]$ \\
\hline 15 & $0.229[2]$ & $0.180[2]$ & $0.161[2]$ & $0.138[2]$ & $0.123[2]$ & $0.111[2]$ & $0.102[2]$ \\
\hline 20 & $0.177[2]$ & $0.129[2]$ & $0.107[2]$ & $0.824[1]$ & $0.679[1]$ & $0.581[1]$ & $0.509[1]$ \\
\hline 25 & $0.134[2]$ & $0.896[1]$ & $0.685[1]$ & $0.470[1]$ & $0.363[1]$ & $0.300[1]$ & $0.259[1]$ \\
\hline 30 & $0.986[1]$ & $0.597[1]$ & $0.419[1]$ & $0.262[1]$ & $0.198[1]$ & $0.166[1]$ & $0.147[1]$ \\
\hline 35 & $0.699[1]$ & $0.380[1]$ & $0.246[1]$ & $0.149[1]$ & $0.119[1]$ & $0.105[1]$ & 0.963 \\
\hline 40 & $0.471[1]$ & $0.228[1]$ & $0.140[1]$ & 0.911 & 0.809 & 0.760 & 0.713 \\
\hline 45 & $0.294[1]$ & $0.126[1]$ & 0.789 & 0.632 & 0.627 & 0.605 & 0.564 \\
\hline 50 & $0.162[1]$ & 0.629 & 0.465 & 0.508 & 0.532 & 0.506 & 0.460 \\
\hline 55 & $0.729^{\circ}$ & 0.290 & 0.332 & 0.463 & 0.476 & 0.435 & 0.382 \\
\hline 60 & 0.221 & 0.175 & 0.324 & 0.457 & 0.437 & 0.378 & 0.319 \\
\hline 65 & $0.516[-1]$ & 0.228 & 0.394 & 0.467 & 0.404 & 0.329 & 0.267 \\
\hline 70 & 0.162 & 0.395 & 0.504 & 0.475 & 0.370 & 0.283 & 0.223 \\
\hline 75 & 0.475 & 0.621 & 0.620 & 0.470 & 0.330 & 0.239 & 0.183 \\
\hline
\end{tabular}


TABLE II. (Continued).

\begin{tabular}{|c|c|c|c|c|c|c|c|}
\hline$E(\mathrm{eV})$ & 50 & 75 & 100 & 150 & 200 & 250 & 300 \\
\hline 80 & 0.902 & 0.853 & 0.713 & 0.445 & 0.282 & 0.195 & 0.149 \\
\hline 85 & $0.135[1]$ & $0.105[1]$ & 0.760 & 0.397 & 0.229 & 0.154 & 0.119 \\
\hline 90 & $0.174[1]$ & $0.116[1]$ & 0.749 & 0.327 & 0.173 & 0.117 & $0.948[-1]$ \\
\hline 95 & $0.199[1]$ & $0.118[1]$ & 0.678 & 0.244 & 0.121 & $0.869[-1]$ & $0.778[-1]$ \\
\hline 100 & $0.208[1]$ & $0.109[1]$ & 0.555 & 0.158 & $0.769[-1]$ & $0.668[-1]$ & $0.691[-1]$ \\
\hline 105 & $0.198[1]$ & 0.921 & 0.400 & $0.818[-1]$ & $0.485[-1]$ & $0.593[-1]$ & $0.697[-1]$ \\
\hline 110 & $0.172[1]$ & 0.693 & 0.239 & $0.286[-1]$ & $0.412[-1]$ & $0.668[-1]$ & $0.804[-1]$ \\
\hline 115 & $0.135[1]$ & 0.449 & 0.103 & $0.113[-1]$ & $0.592[-1]$ & $0.904[-1]$ & 0.101 \\
\hline 120 & $0.925^{\circ}$ & 0.238 & $0.236[-1]$ & $0.401[-1]$ & 0.105 & 0.131 & 0.133 \\
\hline 125 & 0.532 & 0.104 & $0.263[-1]$ & 0.121 & 0.180 & 0.187 & 0.173 \\
\hline 130 & 0.237 & $0.869[-1]$ & $0.130^{\circ}$ & 0.257 & 0.282 & 0.257 & 0.221 \\
\hline 135 & $0.971[-1]$ & 0.210 & 0.343 & 0.444 & 0.408 & 0.339 & 0.276 \\
\hline 140 & 0.145 & 0.482 & 0.662 & 0.676 & 0.552 & 0.429 & 0.334 \\
\hline 145 & 0.387 & 0.889 & $0.107[1]$ & 0.939 & 0.706 & 0.524 & 0.395 \\
\hline 150 & 0.799 & $0.140[1]$ & $0.154[1]$ & $0.122[1]$ & 0.864 & 0.617 & 0.454 \\
\hline 155 & $0.133[1]$ & $0.198[1]$ & $0.204[1]$ & $0.150[1]$ & $0.102[1]$ & 0.706 & 0.509 \\
\hline 160 & $0.192[1]$ & $0.256[1]$ & $0.252[1]$ & $0.176[1]$ & $0.115[1]$ & 0.786 & 0.558 \\
\hline 165 & $0.249[1]$ & $0.309[1]$ & $0.295[1]$ & $0.198[1]$ & $0.127[1]$ & 0.853 & 0.599 \\
\hline 170 & $0.296[1]$ & $0.352[1]$ & $0.329[1]$ & $0.215[1]$ & $0.136[1]$ & 0.903 & 0.630 \\
\hline 175 & $0.327[1]$ & $0.379[1]$ & $0.351[1]$ & $0.226[1]$ & $0.142[1]$ & 0.934 & 0.649 \\
\hline 180 & $0.338[1]$ & $0.388[1]$ & $0.358[1]$ & $0.230[1]$ & $0.144[1]$ & 0.945 & 0.655 \\
\hline$\sigma_{J}$ & $0.333[2]$ & $0.234[2]$ & $0.192[2]$ & $0.150[2]$ & $0.128[2]$ & $0.114[2]$ & $0.103[2]$ \\
\hline
\end{tabular}

"The notation $a[b]$ means $a \times 10^{b}$.

than at higher energies. The reason possibly is that the polarization interaction plays a more significant role at lower energies. It was also noticed that variation of $d$ has a significant effect on the location of the minima and the maxima of the DCS curves and on the value of the DCS at those locations, especially at low energies. Variation of $d$ also affected the DCS in the forward direction at all energies. Hence the DCS curves could be moved up and down near the forward direction by changing the value of $d$ whereas the shape of the DCS curves at higher angles stayed about the same.

Within the range of values of $d$, shown in Table I for various electron-impact energies, the computed DCS curves and the integrated elastic cross sections remain close to the corresponding experimentally measured values. The value of $d$ that, in our judgment, gave the best fitting was used for final computation of differential and integrated elastic cross sections for the scattering of both the electrons as well as the positrons. The DCS curves in the forward direction for positron impact are more sensitive to the value of $d$ than the corresponding curves for electron impact due to the tendency of cancellation between the static and polarization interactions for the positrons. The differential cross sections for the elastic scattering of positrons from argon are shown in Fig. 5. Numerical values of the differential and integrated elastic cross sections for the scattering of electrons and positrons from argon are provided in Tables II and III.

A comparison of the various cross sections calculated here, for electron scattering, with corresponding measured values is given in Figs. $1-4$ and in Tables IV and V. The calculated DCS curves for elastic scattering of electrons from argon are in good agreement with the measured values of Srivastava et al., ${ }^{27}$ Andrick, ${ }^{28}$ DuBois and Rudd, ${ }^{23}$ and Filipovic ${ }^{30}$ at lower energies $(<50 \mathrm{eV})$ and with Vušković and Kurepa, ${ }^{22}$ and DuBois and Rudd ${ }^{23}$ at higher energies $(100 \leq E \leq 200 \mathrm{eV})$. However, the agreement with Srivastava et al. $^{27}$ and Filipovic ${ }^{30}$ at larger angles becomes progressively poorer as the electron-impact energy becomes large. The differential cross sections measured by Williams and Willis ${ }^{19}$ are almost always lower than our calculated cross sections at the minimum points. The integrated elastic cross sections given in Table IV are consistent with the available experimental values except with those of Srivastava et al. ${ }^{27}$ which are lower than our calculated values. The transport cross sections, which represent the moments of $1-(\cos \theta)^{n}$, and which are related to the momentum transfer or diffusion cross section (for $n=1$ ), viscosity and thermal conductivity cross section (for $n=2$ ), etc., ${ }^{74}$ for both electrons and positrons are tabulated in Table $V$. Comparison in Table $V$ of the present momentum transfer cross sections for the electron-argon system with the corresponding experimental values shows reasonable agreement except with the results of Ref. 27. Phase shifts of the seven lowest partial waves $(l=0-6)$ for various impact energies of electrons and positrons are presented in Table VI and compare favorably with the available measured values.

The DCS curves for the elastic scattering of positrons by argon at various energies are shown in Fig. 5. These 
TABLE III. Differential and integrated cross sections $\left(a_{0}^{2} \mathrm{sr}^{-1}\right)$ for elastic scattering of positrons from argon at $E=3-300 \mathrm{eV}$.

\begin{tabular}{|c|c|c|c|c|c|c|c|}
\hline$\overbrace{\theta(\mathrm{deg})} E(\mathrm{eV})$ & 3 & $\overline{5}$ & 10 & 15 & 20 & 30 & 40 \\
\hline 0 & $0.295[1]^{\mathrm{a}}$ & $0.335[1]$ & $0.212[1]$ & $0.263[1]$ & $0.239[1]$ & $0.276[1]$ & $0.302[1]$ \\
\hline 5 & $0.209[1]$ & $0.210[1]$ & $0.114[1]$ & $0.127[1]$ & $0.134[1]$ & $0.167[1]$ & $0.199[1]$ \\
\hline 10 & $0.140[1]$ & $0.122[1]$ & 0.676 & 0.875 & $0.135[1]$ & $0.193[1]$ & $0.252[1]$ \\
\hline 15 & 0.795 & 0.626 & 0.651 & 0.983 & $0.176[1]$ & $0.253[1]$ & $0.325[1]$ \\
\hline 20 & 0.441 & 0.321 & 0.832 & $0.128[1]$ & $0.220[1]$ & $0.299[1]$ & $0.365[1]$ \\
\hline 25 & 0.220 & 0.189 & $0.109[1]$ & $0.160[1]$ & $0.251[1]$ & $0.318[1]$ & $0.364[1]$ \\
\hline 30 & 0.108 & 0.183 & $0.133[1]$ & $0.185[1]$ & $0.263[1]$ & $0.309[1]$ & $0.332[1]$ \\
\hline 35 & $0.795[-1]$ & 0.252 & $0.153[1]$ & $0.198[1]$ & $0.259[1]$ & $0.281[1]$ & $0.284[1]$ \\
\hline 40 & 0.107 & 0.364 & $0.165[1]$ & $0.201[1]$ & $0.242[1]$ & $0.244[1]$ & $0.232[1]$ \\
\hline 45 & 0.170 & 0.488 & $0.171[1]$ & $0.195[1]$ & $0.218[1]$ & $0.204[1]$ & $0.184[1]$ \\
\hline 50 & 0.256 & 0.615 & $0.170[1]$ & $0.182[1]$ & $0.191[1]$ & $0.167[1]$ & $0.143[1]$ \\
\hline 55 & 0.348 & 0.724 & $0.165[1]$ & $0.166[1]$ & $0.164[1]$ & $0.135[1]$ & $0.112[1]$ \\
\hline 60 & 0.445 & 0.819 & $0.156[1]$ & $0.148[1]$ & $0.138[1]$ & $0.109[1]$ & 0.888 \\
\hline 65 & 0.536 & 0.890 & $0.144[1]$ & $0.131[1]$ & $0.116[1]$ & $0.888^{\circ}$ & 0.722 \\
\hline 70 & 0.617 & 0.937 & $0.132[1]$ & $0.114[1]$ & 0.981 & 0.737 & 0.605 \\
\hline 75 & 0.689 & 0.968 & $0.120[1]$ & $0.990^{\circ}$ & 0.833 & 0.626 & 0.523 \\
\hline 80 & 0.747 & 0.976 & $0.108[1]$ & 0.861 & 0.716 & 0.545 & 0.464 \\
\hline 85 & 0.796 & 0.973 & 0.967 & 0.753 & 0.626 & 0.485 & 0.419 \\
\hline 90 & 0.830 & 0.954 & 0.869 & 0.665 & 0.556 & 0.441 & 0.384 \\
\hline 95 & 0.855 & 0.929 & 0.782 & 0.594 & 0.504 & 0.407 & 0.355 \\
\hline 100 & 0.870 & 0.896 & 0.707 & 0.538 & 0.464 & 0.379 & 0.330 \\
\hline 105 & 0.877 & 0.860 & 0.645 & 0.493 & 0.432 & 0.357 & 0.309 \\
\hline 110 & 0.879 & 0.823 & 0.592 & 0.459 & 0.408 & 0.337 & 0.289 \\
\hline 115 & 0.873 & 0.783 & 0.549 & 0.432 & 0.388 & 0.320 & 0.272 \\
\hline 120 & 0.865 & 0.746 & 0.514 & 0.411 & 0.371 & 0.304 & 0.257 \\
\hline 125 & 0.853 & 0.709 & 0.486 & 0.394 & 0.357 & 0.291 & 0.244 \\
\hline 130 & 0.841 & 0.676 & 0.463 & 0.381 & 0.345 & 0.279 & 0.232 \\
\hline 135 & 0.826 & 0.645 & 0.445 & 0.370 & 0.335 & 0.268 & 0.222 \\
\hline 140 & 0.812 & 0.618 & 0.431 & 0.361 & 0.326 & 0.259 & 0.213 \\
\hline 145 & 0.799 & 0.594 & 0.420 & 0.354 & 0.318 & 0.251 & 0.206 \\
\hline 150 & 0.785 & 0.572 & 0.411 & 0.348 & 0.311 & 0.243 & 0.200 \\
\hline 155 & 0.775 & 0.557 & 0.404 & 0.343 & 0.305 & 0.238 & 0.195 \\
\hline 160 & 0.763 & 0.541 & 0.399 & 0.339 & 0.300 & 0.233 & 0.191 \\
\hline 165 & 0.757 & 0.531 & 0.395 & 0.336 & 0.297 & 0.230 & 0.188 \\
\hline 170 & 0.750 & 0.523 & 0.392 & 0.334 & 0.294 & 0.227 & 0.186 \\
\hline 175 & 0.747 & 0.518 & 0.391 & 0.333 & 0.293 & 0.226 & 0.184 \\
\hline 180 & 0.749 & 0.520 & 0.390 & 0.332 & 0.292 & 0.225 & 0.184 \\
\hline$\sigma_{1}$ & $0.842[1]$ & $0.927[1]$ & $0.118[2]$ & $0.113[2]$ & $0.117[2]$ & $0.109[2]$ & $0.104[2]$ \\
\hline$E(\mathrm{eV})$ & so & 75 & 100 & 150 & 200 & 250 & 300 \\
\hline 0 & $0.334[1]$ & $0.365[1]$ & $0.426[1]$ & $0.532[1]$ & $0.623[1]$ & $0.704[1]$ & $0.777[1]$ \\
\hline 5 & $0.219[1]$ & $0.493[1]$ & $0.640[1]$ & $0.903[1]$ & $0.113[2]$ & $0.132[2]$ & $0.149[2]$ \\
\hline 10 & $0.286[1]$ & $0.653[1]$ & $0.809[1]$ & $0.104[2]$ & $0.119[2]$ & $0.129[2]$ & $0.136[2]$ \\
\hline 15 & $0.364[1]$ & $0.680[1]$ & $0.778[1]$ & $0.875[1]$ & $0.901[1]$ & $0.892[1]$ & $0.865[1]$ \\
\hline 20 & $0.396[1]$ & $0.602[1]$ & $0.630[1]$ & $0.618[1]$ & $0.573[1]$ & $0.522[1]$ & $0.473[1]$ \\
\hline 25 & $0.379[1]$ & $0.477[1]$ & $0.459[1]$ & $0.397[1]$ & $0.339[1]$ & $0.292[1]$ & $0.255[1]$ \\
\hline 30 & $0.331[1]$ & $0.352[1]$ & $0.314[1]$ & $0.248[1]$ & $0.202[1]$ & $0.171[1]$ & $0.148[1]$ \\
\hline 35 & $0.270[1]$ & $0.251[1]$ & $0.211[1]$ & $0.159[1]$ & $0.129[1]$ & $0.110[1]$ & 0.958 \\
\hline 40 & $0.211[1]$ & $0.177[1]$ & $0.144[1]$ & $0.109[1]$ & 0.894 & 0.768 & 0.673 \\
\hline 45 & $0.162[1]$ & $0.127[1]$ & $0.104[1]$ & 0.798 & 0.665 & 0.571 & 0.497 \\
\hline 50 & $0.123[1]$ & 0.950 & $0.789^{\circ}$ & 0.620 & 0.517 & 0.439 & 0.377 \\
\hline 55 & 0.947 & 0.746 & 0.632 & 0.500 & 0.411 & 0.344 & 0.292 \\
\hline 60 & 0.751 & 0.612 & 0.524 & 0.411 & 0.333 & 0.275 & 0.230 \\
\hline 65 & 0.616 & 0.519 & 0.445 & 0.343 & 0.273 & 0.222 & 0.184 \\
\hline 70 & 0.524 & 0.450 & 0.383 & 0.289 & 0.226 & 0.182 & 0.150 \\
\hline 75 & 0.459 & 0.395 & 0.332 & 0.245 & 0.190 & 0.152 & 0.124 \\
\hline 80 & 0.410 & 0.350 & 0.290 & 0.211 & 0.161 & 0.128 & 0.104 \\
\hline
\end{tabular}


TABLE III. (Continued).

\begin{tabular}{|c|c|c|c|c|c|c|c|}
\hline$E(\mathrm{eV})$ & 50 & 75 & 100 & 150 & 200 & 250 & 300 \\
\hline 85 & 0.372 & 0.312 & 0.256 & 0.183 & 0.138 & 0.109 & $0.886[-1]$ \\
\hline 90 & 0.340 & 0.280 & 0.227 & 0.160 & 0.120 & $0.943[-1]$ & $0.762[-1]$ \\
\hline 95 & 0.313 & 0.252 & 0.202 & 0.141 & 0.106 & $0.824[-1]$ & $0.664[-1]$ \\
\hline 100 & 0.290 & 0.229 & 0.182 & 0.126 & $0.937[-1]$ & $0.728[-1]$ & $0.584[-1]$ \\
\hline 105 & 0.269 & 0.209 & 0.166 & 0.114 & $0.839[-1]$ & $0.649[-1]$ & $0.520[-1]$ \\
\hline 110 & 0.250 & 0.192 & 0.151 & 0.103 & $0.757[-1]$ & $0.584[-1]$ & $0.467[-1]$ \\
\hline 115 & 0.234 & 0.178 & 0.140 & $0.944[-1]$ & $0.690[-1]$ & $0.530[-1]$ & $0.423[-1]$ \\
\hline 120 & 0.220 & 0.166 & 0.130 & $0.871[-1]$ & $0.633[-1]$ & $0.485[-1]$ & $0.387[-1]$ \\
\hline 125 & 0.208 & 0.156 & 0.121 & $0.808[-1]$ & $0.586[-1]$ & $0.448[-1]$ & $0.357[-1]$ \\
\hline 130 & 0.197 & 0.147 & 0.114 & $0.756[-1]$ & $0.546[-1]$ & $0.417[-1]$ & $0.332[-1]$ \\
\hline 135 & 0.188 & 0.140 & 0.108 & $0.712[-1]$ & $0.513[-1]$ & $0.391[-1]$ & $0.311[-1]$ \\
\hline 140 & 0.180 & 0.133 & 0.103 & $0.675[-1]$ & $0.485[-1]$ & $0.369[-1]$ & $0.293[-1]$ \\
\hline 145 & 0.174 & 0.128 & $0.982[-1]$ & $0.644[-1]$ & $0.462[-1]$ & $0.351[-1]$ & $0.279[-1]$ \\
\hline 150 & 0.168 & 0.124 & $0.946[-1]$ & $0.619[-1]$ & $0.443[-1]$ & $0.336[-1]$ & $0.267[-1]$ \\
\hline 155 & 0.164 & 0.120 & $0.917[-1]$ & $0.598[-1]$ & $0.427[-1]$ & $0.325[-1]$ & $0.258[-1]$ \\
\hline 160 & 0.160 & 0.117 & $0.894[-1]$ & $0.582[-1]$ & $0.415[-1]$ & $0.315[-1]$ & $0.250[-1]$ \\
\hline 165 & 0.158 & 0.115 & $0.877[-1]$ & $0.569[-1]$ & $0.406[-1]$ & $0.308[-1]$ & $0.245[-1]$ \\
\hline 170 & 0.156 & 0.114 & $0.864[-1]$ & $0.561[-1]$ & $0.400[-1]$ & $0.303[-1]$ & $0.241[-1]$ \\
\hline 175 & 0.155 & 0.113 & $0.857[-1]$ & $0.556[-1]$ & $0.396[-1]$ & $0.300[-1]$ & $0.238[-1]$ \\
\hline 180 & 0.155 & 0.113 & $0.855[-1]$ & $0.554[-1]$ & $0.395[-1]$ & $0.299[-1]$ & $0.238[-1]$ \\
\hline$\sigma_{I}$ & $0.973[1]$ & $0.102[2]$ & $0.956[1]$ & $0.861[1]$ & $0.791[1]$ & $0.736[1]$ & $0.690[1]$ \\
\hline
\end{tabular}

The notation $a[b]$ means $a \times 10^{b}$.

curves show a minimum at low energies which shifts toward the forward direction with increasing impact energy. The increasing depth of this minimum on lowering the impact energy suggests the existence of the critical point for the positron-argon system. The critical points represent the points of minimum scattering, where a small change in either the incident projectile energy or the scattering angle is associated with an appreciable increase in the differential scattering cross section. The lowenergy critical points for various positron-rare-gas-atom systems have been predicted by Wadehra et al. ${ }^{75}$ So far the measurements of the angular distributions of elastic

TABLE IV. Comparison of calculated integrated elastic cross sections (in units of $a_{0}^{2}$ ) with the experimental values.

\begin{tabular}{|c|c|c|c|}
\hline Projectile & $\begin{array}{c}E \\
(\mathrm{eV})\end{array}$ & $\begin{array}{c}\text { Present } \\
\text { value of } \sigma_{I}\end{array}$ & Experimental value of $\sigma_{I}$ \\
\hline \multirow[t]{13}{*}{$e^{-}$} & 3 & 20.66 & $\begin{array}{l}20.83(7), 19.48(12), 17.3(13), 20.64(14) \\
20.12(26), 19.65(27), 20.51(28)\end{array}$ \\
\hline & 5 & 39.31 & $\begin{array}{l}33.95(7), 32.1(11), 32.59(12), 30.87(13) \\
36.76(14), 36.09(26), 30.02(27), 34.73(28)\end{array}$ \\
\hline & 10 & 76.33 & $\begin{array}{l}70.75(10), 73.47(11), 74.33(12), 67.54(13), \\
69.3(14), 83.4(26), 64.32(27), 77.29(28)\end{array}$ \\
\hline & 15 & 86.57 & $85.5(26), 75.04(27), 85.48(28)$ \\
\hline & 20 & 72.79 & $\begin{array}{l}71.31(19), 68.4(23), 70.65(26), 44.67(27) \\
71.18(28)\end{array}$ \\
\hline & 30 & 51.08 & $47.21(19)$ \\
\hline & 40 & 39.61 & $32.28(19)$ \\
\hline & 50 & 33.28 & $26.48(19), 25.61(23), 21.8(27)$ \\
\hline & 75 & 23.37 & $14.29(27)$ \\
\hline & 100 & 19.21 & $\begin{array}{l}\text { 18.66(19), } 16.51(20), 17.33(22), 17.1(23), \\
18.04(24), 9.29(27)\end{array}$ \\
\hline & 150 & 15.02 & $11.86(19), 13.21(20), 13.33(22), 14.83(24)$ \\
\hline & 200 & 12.8 & $11.51(18), 9.81(19), 10.9(23), 12.68(24)$ \\
\hline & 300 & 10.32 & $8.74(18), 7.82(19), 8.81(20), 10.19(24)$ \\
\hline \multirow[t]{2}{*}{$e^{+}$} & 3 & 8.42 & $12.57(56), 9.11(58), 8.56(64)$ \\
\hline & 5 & 9.27 & $12.56(56), 10.8(57), 9.68(58), 8.73(64)$ \\
\hline
\end{tabular}

"The notation $a(b)$ for experimental values of $\sigma_{f}$ means the measured value of $a$ taken from Ref. $b$. 
TABLE V. The higher transport cross sections $\sigma^{(n)}$ (in units of $a_{0}^{2}$ ) and comparison of $\sigma^{(1)}$ with the experimental values.

\begin{tabular}{ccccccc}
\hline \hline Projectile & $\begin{array}{c}E \\
(\mathrm{eV})\end{array}$ & $\sigma^{(4)}$ & $\sigma^{(3)}$ & $\sigma^{(2)}$ & \multicolumn{1}{c}{$\sigma^{(1)}$} & $\begin{array}{c}\text { Experimental } \\
\text { momentum transfer, } \sigma^{(1)}\end{array}$ \\
\hline$e^{-}$ & 3 & 18.19 & 19.41 & 15.89 & 16.56 & $16.72(26), 14.65(27), 16.08(28)$ \\
& 5 & 30.45 & 38.97 & 25.98 & 34.67 & $32.45(26), 22.87(27), 29.5(28)$ \\
& 10 & 42.15 & 66.37 & 31.61 & 58.97 & $67.65(26), 53.6(27), 62.4(28)$ \\
& 15 & 40.84 & 58.57 & 28.41 & 49.56 & $51.2(26), 53.6(27), 51.28(28)$ \\
& 20 & 34.83 & 41.82 & 24.44 & 33.65 & $33.66(26), 23.58(27), 34.8(28)$ \\
& 30 & 26.18 & 26.52 & 19.40 & 21.33 & $13.22(27)$ \\
& 40 & 20.20 & 20.82 & 15.38 & 17.49 & \\
& 50 & 16.12 & 18.05 & 12.38 & 15.67 & $8.58(27)$ \\
& 75 & 9.98 & 13.40 & 7.60 & 11.90 & $6.79(27)$ \\
& 100 & 7.39 & 11.03 & 5.48 & 9.78 & $5.72(27)$ \\
& 150 & 5.24 & 7.96 & 3.74 & 6.95 & \\
& 200 & 4.26 & 6.08 & 2.99 & 5.22 & \\
& 250 & 3.63 & 4.86 & 2.53 & 4.09 & \\
& 300 & 3.16 & 4.01 & 2.19 & 3.31 & \\
3 & 6.87 & 9.02 & 5.89 & 9.71 & \\
& 5 & 7.87 & 9.39 & 6.80 & 9.44 & \\
& 10 & 9.67 & 10.40 & 8.01 & 8.95 & \\
& 15 & 8.85 & 9.33 & 7.15 & 7.64 & \\
20 & 8.63 & 8.92 & 6.79 & 7.01 & \\
& 30 & 7.51 & 7.62 & 5.78 & 5.79 & \\
40 & 6.73 & 6.73 & 5.10 & 5.00 & \\
50 & 6.04 & 5.98 & 4.55 & 4.38 & \\
75 & 5.38 & 5.19 & 3.95 & 3.63 & \\
& 100 & 4.62 & 4.40 & 3.36 & 2.30 & \\
150 & 3.64 & 3.40 & 2.60 & 2.22 & \\
200 & 3.01 & 2.77 & 2.12 & 1.74 & \\
& 250 & 2.56 & 2.33 & 1.77 & 1.43 & \\
300 & 2.22 & 2.00 & 1.52 & 1.20 & \\
& & &
\end{tabular}

The notation $a(b)$ for experimental values of $\sigma^{(1)}$ means the measured value of $a$ taken from Ref. $b$.

scattering of positrons by argon have been made only for a limited range of energies. The only available measured relative values of differential cross sections of Hyder et $a l^{3}$ at energies 100,200 , and $300 \mathrm{eV}$, have been normalized to the present calculated DCS curves at $90^{\circ}$. In this energy range, other calculations of elastic cross sections are those of McEachran and Stauffer ${ }^{69}$ using the polarized orbital method and some limited results by Joachain ${ }^{47}$ using the optical model potential. At higher energies and at larger angles the present calculations of DCS agree with those of McEachran and Stauffer and with the measurements of Hyder et al. When normalized separately at $90^{\circ}$, the measurements of Hyder et al. at 300 $\mathrm{eV}$ agree well both with the present calculation as well as with the calculations of Joachain. ${ }^{47}$ At low energies and near the forward scattering direction, where unfortunately no experimental information for positron-argon elastic scattering is available, our DCS curves differ significantly from the calculated results of McEachran and Stauffer. It would certainly be worthwhile to carry out experiments on the elastic differential scattering of positrons by argon in this energy and angular range. For positron energies smaller than the positronium formation threshold in Ar $(8.96 \mathrm{eV})$, the integrated elastic cross sections are compared in Table IV with the measured total positron-argon collisional cross sections. Higher transport cross sections and the lowest seven phase shifts for positron-argon elastic scattering are given in Tables $V$ and VI, respectively. No comparison of these numbers is possible due to the lack of measurements of these quantities.

Finally, an attempt was made to obtain the cross sections for the elastic scattering of ultralow $(\leq 2.5 \mathrm{eV})$ energy electrons and positrons by argon. In this energy range, no experimental numerical values of differential cross sections for electron impact are available. Thus it was not possible to obtain the low-energy parameters such as the scattering length and effective range for the positronargon system using the present procedure.

In the present paper we have obtained various cross sections-differential, integrated, momentum transfer, etc.- and the corresponding phase shifts for the elastic scattering of positrons and electrons by argon. These cross sections compare favorably with the recent measurements of elastic differential scattering of intermediateenergy positrons by argon. With the anticipation that similar measurements will be made for other rare-gas targets in the near future, we are presently calculating the cross sections for the elastic scattering of low- and intermediate-energy positrons by $\mathrm{He}, \mathrm{Ne}, \mathrm{Kr}$, and $\mathrm{Xe}$ by a similar procedure. 
TABLE VI. Phase shifts (rad) of the first seven partial waves for elastic scattering of electrons and positrons by argon.

\begin{tabular}{|c|c|c|c|c|c|c|c|c|}
\hline Projectile & $E(\mathrm{eV})$ & $l=0$ & $l=1$ & $l=2$ & $l=3$ & $l=4$ & $l=5$ & $l=6$ \\
\hline \multirow[t]{29}{*}{$e^{-}$} & 3 & -0.490 & -0.132 & 0.130 & 0.0241 & 0.0106 & 0.0057 & 0.00346 \\
\hline & & -0.457 & -0.134 & 0.142 & $0.021(26)^{2}$ & & & \\
\hline & & -0.548 & -0.140 & 0.125 & $0.035(27)$ & & & \\
\hline & & -0.493 & -0.142 & 0.120 & $0.025(28)$ & & & \\
\hline & 5 & -0.747 & -0.272 & 0.306 & 0.042 & 0.0175 & 0.0094 & 0.0057 \\
\hline & & -0.685 & -0.205 & 0.317 & $0.031(26)$ & & & \\
\hline & & -0.747 & -0.256 & 0.254 & $0.102(27)$ & & & \\
\hline & & -0.733 & -0.277 & 0.260 & $0.044(28)$ & & & \\
\hline & 10 & -1.283 & -0.688 & 0.751 & 0.084 & 0.0321 & 0.0172 & 0.0106 \\
\hline & & -1.098 & -0.528 & 0.936 & $0.093(26)$ & & & \\
\hline & & -1.243 & -0.430 & 0.805 & $0.171(27)$ & & & \\
\hline & & -1.143 & -0.562 & 0.840 & $0.10(28)$ & & & \\
\hline & 15 & -1.569 & -0.867 & 1.131 & 0.140 & 0.0497 & 0.0255 & 0.0156 \\
\hline & & -1.394 & -0.750 & 1.451 & $0.154(26)$ & & & \\
\hline & & -1.365 & -0.506 & 1.593 & $0.2(27)$ & & & \\
\hline & & -1.443 & -0.782 & 1.39 & $0.165(28)$ & & & \\
\hline & 20 & -1.826 & -1.064 & 1.519 & 0.186 & 0.064 & 0.032 & 0.0194 \\
\hline & & -1.653 & -0.935 & 1.747 & $0.241(26)$ & & & \\
\hline & & -1.818 & -0.871 & 1.679 & $0.262(27)$ & & & \\
\hline & & -1.683 & -0.962 & 1.670 & $0.232(28)$ & & & \\
\hline & 30 & -2.154 & -1.296 & 1.817 & 0.306 & 0.104 & 0.0496 & 0.0288 \\
\hline & 40 & -2.40 & -1.47 & 1.929 & 0.421 & 0.147 & 0.0682 & 0.0386 \\
\hline & 50 & -2.583 & -1.595 & 2.008 & 0.539 & 0.196 & 0.0909 & 0.0503 \\
\hline & 75 & 0.122 & 1.207 & -1.184 & 0.645 & 0.250 & 0.116 & 0.0622 \\
\hline & 100 & -0.146 & 1.014 & -1.166 & 0.773 & 0.330 & 0.159 & 0.0858 \\
\hline & 150 & -0.532 & 0.735 & -1.168 & 0.929 & 0.460 & 0.241 & 0.135 \\
\hline & 200 & -0.809 & 0.535 & -1.185 & 1.013 & 0.552 & 0.311 & 0.182 \\
\hline & 250 & -1.027 & 0.38 & -1.207 & 1.064 & 0.619 & 0.368 & 0.225 \\
\hline & 300 & -1.205 & 0.253 & -1.229 & 1.096 & 0.668 & 0.415 & 0.263 \\
\hline \multirow[t]{14}{*}{$e^{+}$} & 3 & -0.334 & 0.0839 & 0.0528 & 0.0219 & 0.0105 & 0.00572 & 0.00346 \\
\hline & 5 & -0.496 & 0.0505 & 0.0703 & 0.0341 & 0.0169 & 0.00939 & 0.00572 \\
\hline & 10 & -0.878 & -0.139 & 0.0455 & 0.0456 & 0.0278 & 0.0167 & 0.0106 \\
\hline & 15 & -1.06 & -0.251 & 0.0173 & 0.0509 & 0.0368 & 0.0235 & 0.0152 \\
\hline & 20 & -1.245 & -0.384 & -0.0427 & 0.0371 & 0.0378 & 0.027 & 0.0185 \\
\hline & 30 & -1.455 & -0.545 & -0.126 & 0.0132 & 0.0385 & 0.0335 & 0.0248 \\
\hline & 40 & 1.529 & -0.674 & -0.206 & -2.081 & 0.0306 & 0.0354 & 0.0291 \\
\hline & 50 & 1.423 & -0.765 & -0.267 & -0.0506 & 0.0217 & 0.036 & 0.0326 \\
\hline & 75 & 1.115 & -1.044 & -0.487 & -0.196 & -0.0598 & -0.0038 & 0.0154 \\
\hline & 100 & 0.967 & -1.185 & -0.606 & -0.284 & -0.115 & -0.0344 & 0.00064 \\
\hline & 150 & 0.789 & -1.365 & -0.774 & -0.420 & -0.214 & -0.986 & -0.0374 \\
\hline & 200 & 0.690 & -1.474 & -0.884 & -0.519 & -0.294 & -0.158 & -0.778 \\
\hline & 250 & 0.631 & -1.543 & -0.960 & -0.593 & -0.359 & -0.209 & -0.116 \\
\hline & 300 & 0.596 & 1.552 & -1.015 & -0.651 & -0.412 & -0.254 & -0.151 \\
\hline
\end{tabular}

Numbers in parentheses denote reference from which the measured phase shift was taken.

\section{ACKNOWLEDGMENTS}

We thank Dr. D. Andrick, Dr. A. K. Bhatia, Dr. D. Filipović, Dr. W. E. Kauppila, Dr. A. D. Stauffer, and Dr. T. S. Stein for providing unpublished data. Support of the National Science Foundation (Grant No. PHY 8311705) and the Air Force Office of Scientific Research (Grant No. AFOSR-84-0143) is gratefully acknowledged.

\section{APPENDIX A: GENERATION OF SPHERICAL BESSEL FUNCTIONS}

The standard recursion relations ${ }^{76}$ can be used for the generation of the spherical Bessel functions $n_{l}(x)$ and $k_{l}(x)$ for any value of the argument $x$ and for increasing $l$ as well as for the generation of $j_{l}(x)$ for increasing $l$ and for argument $x>50$. However, due to a numerical instability, these recurrence relations cannot be used for the generation of $j_{l}(x)$ for increasing $l$ if $x \leq 50$ and for the generation of $i_{l}(x)$ for increasing $l$ for any value of the argument $x$. In these cases $i_{l}(x)$ and $j_{l}(x)$ can be evaluated using hypergeometric function of $F_{1}$ :

$i_{l}(x)=\sqrt{\pi / 2 x}(x / 2)^{l+1 / 2}{ }_{0} F_{1}\left(l+\frac{3}{2} ; x^{2} / 4\right) / \Gamma\left(l+\frac{3}{2}\right)$,

$j_{l}(x)=\sqrt{\pi / 2 x}(x / 2)^{l+1 / 2}{ }_{0} F_{1}\left(l+\frac{3}{2} ;-x^{2} / 4\right) / \Gamma\left(l+\frac{3}{2}\right)$. 
The function ${ }_{0} F_{1}$ can be evaluated using the rational approximation. ${ }^{77}$ When the argument $x$ of $j_{l}$ is large (>50), the rational approximation for ${ }_{0} F_{1}$ becomes unstable.

\section{APPENDIX B: DERIVATION OF PHASE SHIFT FOR POLARIZATION POTENTIAL IN BORN APPROXIMATION}

For the polarization potential, Eq. (18), the Born phase-shift integral $T_{B l}$, Eq. (5), is defined to be $T_{p B l}$ and is given by

$$
T_{p B l}=\alpha k \int_{0}^{\infty} r^{4} j_{l}^{2}(k r) /\left(r^{2}+d^{2}\right)^{3} d r .
$$

After some simplifications, Eq. (B1) can be written as

$$
T_{p B l}=\alpha k^{2}\left[\int_{0}^{\infty} x^{2} j_{l}^{2}(x) /\left(x^{2}+a^{2}\right)^{2} d x-a^{2} \int_{0}^{\infty} x^{2} j_{l}^{2}(x) /\left(x^{2}+a^{2}\right)^{3} d x\right],
$$

where $a=k d$. Using Eq. (6.535) of Ref. 78 and the recurrence relations for the spherical Bessel functions $i_{l}(x)$ and $k_{l}(x)$ one can derive

$$
\begin{aligned}
\int_{0}^{\infty} x^{2} j_{l}^{2}(x) /\left(x^{2}+a^{2}\right)^{2} d x= & -\left[2 a i_{l+1}(a) k_{l}(a)-\pi /(2 a)+(2 l+1) i_{l}(a) k_{l}(a)\right] / 2(a) \\
\int_{0}^{\infty} x^{2} j_{l}^{2}(x) /\left(x^{2}+a^{2}\right)^{3} d x=- & {\left[2 a i_{l+1}(a) k_{l}(a)-\pi /(2 a)+(2 l+1) i_{l}(a) k_{l}(a)\right] /\left(8 a^{3}\right) } \\
+ & \left\{4 l_{l+1}(a) k_{l}(a)-\pi l / a^{2}+i_{l}(a) k_{l}(a) / a\left[2 a^{2}+4\left(l+\frac{1}{2}\right)^{2}-2 l-1\right]\right. \\
& \left.-2 a i_{l+1}(a) k_{l+1}(a)\right\} /\left(8 a^{2}\right) .
\end{aligned}
$$

Substituting (B3) and (B4) in (B2) and after some simplification Eq. (20) is obtained.

\section{APPENDIX C: DERIVATION OF PHASE SHIFT FOR STATIC POTENTIAL IN BORN APPROXIMATION}

Substituting $V_{s}(r)[\mathrm{Eq} .(17)]$, in Eq. (5) and making use of the standard integral ${ }^{78}$

$$
\int_{0}^{\infty} r \exp (-z r) j_{l}^{2}(k r) d r=\left(2 k^{2}\right)^{-1} Q_{l}\left[1+z^{2} /\left(2 k^{2}\right)\right] \text {, }
$$

where $Q_{l}$ is the Legendre function of the second kind, Eq. (22) is obtained. The argument of the $Q_{l}$ function, $1+z^{2} /\left(2 k^{2}\right)$, is greater than 1 and therefore the recurrence relation cannot be used for the generation of the functions for increasing $l$. One convenient way to evaluate a $Q_{l}$ function is to write it in terms of Gaussian hypergeometric function $F(a, b ; c ; x)$ as $^{76}$

$$
Q_{l}(x)=\frac{\pi^{1 / 2} \Gamma(l+1)}{2^{l+1} \Gamma\left(l+\frac{3}{2}\right)} \frac{1}{x^{l+1}} F\left(1+l / 2, \frac{1}{2}+l / 2 ; l+\frac{3}{2} ; 1 / x^{2}\right), x>1
$$

where the hypergeometric function can be evaluated by summing the following series:

$$
F(a, b ; c ; x)=1+\frac{a b}{c \times 1} x+\frac{a(a+1) b(b+1)}{c(c+1) \times 1 \times 2} x^{2}+\frac{a(a+1)(a+2) b(b+1)(b+2)}{c(c+1)(c+2) \times 1 \times 2 \times 3} x^{3}+\cdots .
$$

The higher-order derivatives of $Q_{l}$ with respect to $z$ can be carried out easily using Eq. (C2) and the relation

$$
\frac{d^{n}}{d x^{n}} F(a, b ; c ; x)=\frac{\Gamma(a+n) \Gamma(b+n) \Gamma(c)}{\Gamma(c+n) \Gamma(a) \Gamma(b)} F(a+n, b+n ; c+n ; x) \text {. }
$$

1C. Ramsauer, Ann. Phys. (Leipzig) 66, 546 (1921).

2W. E. Kauppila and T. S. Stein, Can. J. Phys. 60, 471 (1982); T. S. Stein and W. E. Kauppila, Adv. At. Mol. Phys. 18, 53 (1982).

${ }^{3}$ G. M. A. Hyder, M. S. Dababneh, Y.-F. Hsieh, W. E. Kauppila, C. K. Kwan, M. Mahdavi-Hezaveh, and T. S. Stein, Phys. Rev. Lett. 57, 2252 (1986).

${ }^{4}$ G. M. Webb, Phys. Rev. 47, 379 (1935).

${ }^{5}$ W. Aberth, G. Sunshine, and B. Bederson, Abstracts of the Third International Conference on the Physics of Electronic and Atomic Collisions, London, 1963, edited by M. R. C. McDowell (North-Holland, Amsterdam, 1964), p. 53.
6D. E. Golden and H. W. Bandel, Phys. Rev. 149, 58 (1966)

7W. E. Kauppila, T. S. Stein, G. Jesion, M. S. Dababneh, and V. Pol, Rev. Sci. Instrum. 48, 822 (1977).

${ }^{8}$ R. W. Wagenaar and F. J. de Heer, J. Phys. B 13, 3855 (1980).

${ }^{9}$ R. W. Wagenaar and F. J. de Heer, J. Phys. B 18, 2021 (1985).

10W. E. Kauppila, T. S. Stein, J. H. Smart, M. S. Dababneh, Y. K. Ho, J. P. Downing, and V. Pol, Phys. Rev. A 24, 725 (1981).

11J. C. Nickel, 1. Imre, D. F. Register, and S. Trajmar, J. Phys. B 18, 125 (1985).

${ }^{12}$ K. Jost, P. G. F. Bisling, F. Eschen, M. Felsmann, and L. Walther, in Abstracts of Contributed Papers, Thirteenth Inter- 
national Conference on the Physics of Electronic and Atomic Collisions, Berlin, 1983, edited by J. Eichler, W. Fritsch, I. V. Hertel, N. Stolterfoht, and W. Willie (North-Holland, Amsterdam, 1983), p. 91; A. K. Bhatia (private communication).

13J. Ferch, B. Granitza, C. Masche, and W. Raith, J. Phys. B 18,967 (1985).

${ }^{14}$ M. Charlton, T. C. Griffith, G. R. Heyland, and T. R. Twomey, J. Phys. B 13, L239 (1980).

${ }^{15}$ Yu. K. Gus'kov, R. V. Savvov, and V. A. Slobodyanyuk, Zh. Tekh. Fiz. 48, 277 (1978) [Sov. Phys.-Tech. Phys. 23, 167 (1978)].

16]. Mehr, Z. Phys. 198, 345 (1967).

${ }^{17}$ K. Schackert, Z. Phys. 213, 316 (1968).

18J. P. Bromberg, J. Chem. Phys. 61, 963 (1974).

19J. F. Williams and B. A. Willis, J. Phys. B 8, 1670 (1975).

${ }^{20}$ R. H. J. Jansen, F. J. de Heer, H. J. Luyken, B. van Wingerden, and H. J. Blaauw, J. Phys. B 9, 185 (1976).

${ }^{21}$ B. R. Lewis, J. B. Furness, P. J. O. Teubner, and E. Weigold, J. Phys. B 7, 1083 (1974).

22L. Vušković and M. V. Kurepa, J. Phys. B 9, 837 (1976).

${ }^{23}$ R. D. DuBois and M. E. Rudd, J. Phys. B 9, 2657 (1976).

${ }^{24}$ S. C. Gupta, Ph.D. thesis, University of Liverpool, 1975 (the data are quoted in Ref. 31).

25S. C. Gupta and J. A. Rees, J. Phys. B 8, 1267 (1975).

26J. F. Williams, J. Phys. B 12, 265 (1979).

${ }^{27}$ S. K. Srivastava, H. Tanaka, A. Chutjian, and S. Trajmar, Phys. Rev. A 23, 2156 (1981).

28D. Andrick (private communication).

${ }^{29}$ Zhou Qing, M. J. M. Beerlage, and M. J. van der Weil, Physica C 113, 225 (1982).

30Dusan M. Filipović, M. Sci. thesis, Institute of Physics, Beograd, 1984.

${ }^{31}$ F. J. de Heer, R. H. J. Jansen, and W. van der Kaay, J. Phys. B 12, 979 (1979).

32D. A. McPherson, R. K. Feeney, and J. W. Hooper, Phys. Rev, A 13, 167 (1976).

${ }^{33}$ K. S. Golovanivsky and A. P. Kabilan, Phys. Lett. 80A, 249 (1980).

${ }^{34}$ G. N. Haddad and T. F. O'Malley, Aust. J. Phys. 35, 35 (1982).

${ }^{35}$ D. W. Walker, Adv. Phys. 20, 257 (1971).

${ }^{36}$ M. Fink and A. C. Yates, At. Data 1, 385 (1970).

${ }^{37}$ D. G. Thompson, Proc. R. Soc. London, Ser. A 294, 160 (1966); J. Phys. B 4, 468 (1971).

${ }^{38}$ A. Garbaty and R. W. LaBahn, Phys. Rev. A 4, 1425 (1971).

${ }^{39}$ A. W. Yau, R. P. McEachran, and A. D. Stauffer, J. Phys. B 11,2907 (1978).

${ }^{40}$ A. Dasgupta and A. K. Bhatia, Phys. Rev. A 32, 3335 (1985).

${ }^{41}$ W. C. Fon, K. A. Berrington, P. G. Burke, and A. Hibbert, J. Phys. B 16, 307 (1983).

${ }^{42}$ K. L. Bell, N. S. Scott, and M. A. Lennon, J. Phys. B 17, 4757 (1984).

${ }^{43}$ M. S. Pindzola and H. P. Kelly, Phys. Rev. A 9, 323 (1974).

${ }^{44}$ M. Ya. Amusia, N. A. Cherepkov, L. V. Chernysheva, D. M. Davidović, and V. Radojević, Phys. Rev. A 25, 219 (1982).

${ }^{45}$ I. E. McCarthy, C. J. Noble, B. A. Phillips, and A. D. Turnbull, Phys. Rev. A 15, 2173 (1977).

${ }^{46}$ C. J. Joachain, R. Vanderpoorten, K. H. Winters, and F. W. Byron, Jr., J. Phys. B 10, 227 (1977).

${ }^{47} \mathrm{C}$. J. Joachain, Proceedings of the Tenth International Conference on the Physics of Electronic and Atomic Collisions, Paris, 1977, edited by G. Watel (North-Holland, Amsterdam, 1977), p. 71.

${ }^{48} \mathrm{G}$. Staszewska, D. W. Schwenke, and D. G. Truhlar, Phys.
Rev. A 29, 3078 (1984).

${ }^{49}$ R. A. Berg, J. E. Purcell, and A. E. S. Green, Phys. Rev. A 3, 508 (1971).

50S. K. Datta, S. K. Mandal, P. Khan, and A. S. Ghosh, in Proceedings of the Seventh International Conference on Positron Annihilation (ICPA), edited by P. C. Jain, R. M. Singru, and K. P. Gopinathan (World Scientific, Singapore, 1985), p. 387; Phys. Rev. A 32, 633 (1985).

5is. P. Khare and P. Shobha, J. Phys. B 7, 420 (1974).

${ }^{52}$ R. P. McEachran and A. D. Stauffer, J. Phys. B 16, 4023 (1983).

${ }^{53}$ R. Haberland, L. Fritsche, and J. Noffke, Phys. Rev. A 33, 2305 (1986).

S4L. S. Frost and A. V. Phelps, Phys. Rev. 136, A1538 (1964).

${ }^{55}$ H. B. Milloy, R. W. Crompton, J. A. Rees, and A. G. Robertson, Aust. J. Phys. 30, 61 (1977).

${ }^{56}$ K. F. Canter, P. G. Coleman, T. C. Griffith, and G. R. Heyland, Appl. Phys. 3, 249 (1974).

57B. Jaduszliwer and D. A. Paul, Can. J. Phys. 52, 272 (1974); T. S. Stein (private communication).

${ }^{58}$ W. E. Kauppila, T. S. Stein, and G. Jesion, Phys. Rev. Lett. $36,580(1976)$

${ }^{59}$ P. G. Coleman, T. C. Griffith, G. R. Heyland, and T. R. Twomey, Appl. Phys. 11, 321 (1976).

${ }^{60} \mathrm{~T}$. C. Griffith, G. R. Heyland, and T. R. Twomey, as reported by T. C. Griffith and G. R. Heyland, Phys. Rep. 39, 169 (1978).

61J-S. Tsai, L. Lebow, and D. A. L. Paul, Can. J. Phys, 54, 1741 (1976).

${ }^{62}$ A. G. Brenton, J. Dutton, and F. M. Harris, J. Phys. B 11, L15 (1978)

${ }^{63}$ G. Sinapius, W. Raith, and W. G. Wilson, J. Phys. B 13, 4079 (1980).

${ }^{64}$ P. G. Coleman, J. D. McNutt, L. M. Diana, and J. T. Hutton, Phys. Rev. A 22, 2290 (1980); T. S. Stein (private communication).

${ }^{65}$ T. S. Stein and W. E. Kauppila, in Proceedings of the Fourteenth International Conference on the Physics of Electronic and Atomic Collisions, edited by D. C. Lorents, W. E. Meyerhof, and J. R. Peterson (North-Holland, Amsterdam, 1986), p. 105.

66P. G. Coleman and J. D. MeNutt, Phys. Rev. Lett. 42, 1130 (1979).

67S. P. Khare, A. Kumar, and K. Lata, Indian J. Pure Appl. Phys. 20, 379 (1982); Phys. Rev. A 33, 2795 (1986).

${ }^{68}$ R. P. McEachran, A. G. Ryman, and A. D. Stauffer, J. Phys. B 12, 1031 (1979).

${ }^{69}$ R. P. McEachran and A. D. Stauffer, in Proceedings of the Third International Workshop on Positron (Electron)-Gas Scattering, edited by W. E. Kauppila, T. S. Stein, and J. M. Wadehra (World Scientific, Singapore, 1986), p. 122.

${ }^{70} \mathrm{P}$. U. Arifov and G. I. Zhuravleva, in Proceedings of the Seventh International Conference on Positron Annihilation (ICPA), edited by P. C. Jain, R. M. Singru, and K. P. Gopinathan (World Scientific, Singapore, 1985), p. 425.

7'E. Clementi and C. Roetti, At. Data Nucl. Data Tables 14, 177 (1974).

${ }^{72}$ B. L. Jhanwar and S. P. Khare, Phys. Lett. SOA, 201 (1974).

${ }^{73}$ M. E. Riley and D. G. Truhlar, J. Chem. Phys. 63, 2182 (1975); J. B. Furness and I. E. McCarthy, J. Phys. B 6, 2280 (1973); B. H. Bransden, M. R. C. McDowell, C. J. Noble, and T. Scott, ibid. 9, 1301 (1976).

${ }^{74} \mathrm{See}$, for example, E. W. McDaniel and E. A. Mason, The Mobility and Diffusion of Ions in Gases (Wiley, New York, 1973). 
75J. M. Wadehra, T. S. Stein, and W. E. Kauppila, Phys. Rev. A 29, 2912 (1984).

${ }^{76} \mathrm{See}$, for example, Handbook of Mathematical Functions, edited by M. Abramowitz and I. A. Stegun (Dover, New York, 1970).

${ }^{77}$ See, for example, Yudell L. Luke, Algorithms for the Compu- tation of Mathematical Functions (Academic, New York, 1977).

${ }^{78}$ See, for example, Table of Integrals, Series, and Products, edited by I. S. Gradshteyn and I. M. Ryzhik (Academic, New York, 1980). 